Original article

\title{
Early life adversity blunts responses to pioglitazone in depressed, overweight adults
}

\author{
Thalia K. Robakis ${ }^{\mathrm{a}, *}$, Kathleen Watson-Lin ${ }^{\mathrm{a}}$, Tonita E. Wroolie ${ }^{\mathrm{a}}$, Alison Myoraku ${ }^{\mathrm{a}}$, \\ Carla Nasca $^{\mathrm{b}}$, Benedetta Bigio ${ }^{\mathrm{b}}$, Bruce McEwen ${ }^{\mathrm{b}}$, Natalie L. Rasgon ${ }^{\mathrm{a}}$ \\ a Stanford University, Department of Psychiatry and Behavioral Sciences, United States \\ ${ }^{\mathrm{b}}$ Laboratory of Neuroendocrinology, The Rockefeller University, 1230 York Ave, NY, NY, 10065, United States
}

\section{A R T I C L E I N F O}

\section{Article history:}

Received 15 August 2018

Received in revised form 26 September 2018

Accepted 28 September 2018

Available online 29 October 2018

\section{Keywords:}

Unipolar depression

Neuroendocrinology

Other psychopharmacology

\begin{abstract}
A B S T R A C T
Purpose: Early life adversity is associated with both metabolic impairment and depression in adulthood, as well as with poorer responses to antidepressant medications. It is not yet known whether individual differences in sensitivity to antidiabetic medications could also be related to early life adversity. We examined whether a history of early life adversity affected the observed changes in metabolic function and depressive symptoms in a randomized trial of pioglitazone for augmentation of standard treatments for depression.

Purpose: Early life adversity is associated with both metabolic impairment and depression in adulthood, as well as with poorer responses to antidepressant medications. It is not yet known whether individual differences in sensitivity to antidiabetic medications could also be related to early life adversity. We examined whether a history of early life adversity affected the observed changes in metabolic function and depressive symptoms in a randomized trial of pioglitazone for augmentation of standard treatments for depression.

Findings: We found that early life adversity significantly impaired the metabolic response to pioglitazone. Effects on depressive symptoms did not reach significance, but nonetheless suggested that pioglitazone could mitigate the depressant effects of childhood adversity, only among those insulin resistant at baseline.

Conclusions: We conclude that a history of early life adversity may impair the body's ability to respond to insulin sensitizing pharmacotherapy, and furthermore that its contribution to resistant depression may function in part via the generation of an insulin resistant phenotype.
\end{abstract}

(c) 2018 Elsevier Masson SAS. All rights reserved.

\section{Introduction}

A wealth of evidence indicates that adverse experiences in early life can predispose to metabolic syndrome and diabetes in adulthood [1], as well as to depressive disorders [2]. Depression and metabolic impairment are themselves interrelated [3], suggesting that childhood adversity may trigger a complex cascade of physiological events that interact continuously over the lifespan to alter both physical and mental health [4].

Less is known about the ways in which a history of early life adversity may affect the body's response to existing insulin sensitizing treatments. Individuals with diabetes vary in their responsiveness to available antidiabetic treatments, even when

\footnotetext{
* Corresponding author at: Stanford University, Department of Psychiatry and Behavioral Sciences, 401 Quarry Road, MC 5723, Stanford, CA, 94305, United States. E-mail address: trobakis@stanford.edu (T.K. Robakis).
}

treatments are adhered to appropriately [5]. Sustained control of serum blood glucose is the most important factor in mitigating the long-term health consequences of living with diabetes [6]. Thus, information about conditions that predispose individuals to be resistant to metabolic sensitizers is of clinical importance.

Lack of response to antidepressant medication is also an area of clinical concern [7], and it is known that early life adversity can impair response to treatments for depression [8]. It has also been suggested that metabolic impairment may contribute to treatment resistant depression, possibly via proinflammatory effects [9]. Insulin sensitizing medications have shown promise in improving response to standard treatments for depression [10-13].

Pioglitazone is a PPAR- $\gamma$ agonist in routine use in clinical care. It improves metabolic function via a diversity of mechanisms, including regulation of lipid metabolism, induction of mitochondrial uncoupling, inhibition of cortisol production, and downregulation of proinflammatory cytokines [14]. It has also been investigated for weight loss in obese, nondiabetic individuals, and found to reduce visceral fat [15] and favor 
redistribution of fat to lower body depots, which are not associated with increased cardiovascular risk [16].

Our group has previously investigated the use of pioglitazone as an augmenting agent for depression in a randomized trial. Results from this trial [13] indicated that pioglitazone treatment improved the response to usual care for depression among depressed, insulin-resistant individuals. This suggested that ability to respond to treatments for depression could be impaired by the presence of insulin resistance, and that this barrier could be overcome by treatment with pioglitazone.

Because both insulin resistance and chronic depression are known to be adult sequelae of childhood adversity [1,2], we wished to investigate whether a history of early life adversity would affect response to treatment with pioglitazone. Specifically, we hypothesized that a history of early life adversity could engender a medicationresistant metabolic phenotype, such that individuals with histories of childhood adversity would see more moderate responses to treatment with pioglitazone than those without such histories.

\section{Aims and hypotheses}

Our first aim was to determine whether a history of childhood adversity would affect response to pioglitazone. We predicted that a history of early life adversity (measured by score on the Childhood Trauma Questionnaire, CTQ) would interact with study group assignment (pioglitazone or placebo) in affecting the changes in metabolic parameters and depressive symptoms observed over the twelve weeks of the study.

Our second aim was to determine whether, among individuals treated with pioglitazone, those with histories of early life adversity would be more resistant to the beneficial effects of pioglitazone on both a) depressive symptoms and b) metabolic parameters. We predicted that higher CTQ scores would correlate with smaller observed reductions in depressive symptoms, glucose, insulin, and HOMA among individuals exposed to pioglitazone.

\section{Methods}

Methodology for this trial has been reported elsewhere [13]. Briefly, nondiabetic individuals between 21 and 75 years old, having a body mass index (BMI) of $18.5-40 \mathrm{~kg} / \mathrm{m}^{2}$, having at least 12 years of education, and having a history of depression with at least 8 weeks of stable TAU for depression, were recruited and randomized to receive twelve weeks of treatment with either placebo or $30 \mathrm{mg} /$ day of pioglitazone. Forty-three subjects were recruited, of whom 38 completed the study: 21 in the pioglitazone group and 17 in the placebo group. Of the five dropouts, two were receiving placebo and three active drug. All subjects completed a CTQ. The CTQ is a 28-item self-report questionnaire that assesses exposure to five subtypes of early life adversity [17]. Fasting plasma insulin and glucose as well as a two-hour oral glucose tolerance test (OGTT) were obtained at the beginning and end of the study. HOMA was calculated as (fasting glucose ${ }^{*}$ fasting insulin)/405. Participants were categorized as insulin resistant if any of the following applied: baseline fasting glucose over 100, baseline fasting insulin over 15 , or serum glucose of over 140 at $120 \mathrm{~min}$ post initial glucose challenge. Symptoms of depression were assessed with the Hamilton rating scale for depression (HAM-D) every two weeks for the duration of the study.

\subsection{Statistics}

For Aim 1, we used paired-sample $\mathrm{T}$ tests to confirm that pioglitazone was effective in improving metabolic parameters. We then used univariate ANCOVAs to look for associations between response to pioglitazone treatment (as measured by insulin, glucose, HOMA, OGTT, and HDRS-21) and early life adversity (CTQ total and each of five subscales).

For Aim 2, we plotted the associations between childhood adversity variables and changes in metabolic variables to observe their direction.

\section{Results}

4.1. Childhood adversity and pioglitazone treatment interact to effect changes in metabolism over time

Clinical characteristics of the sample are displayed in Table 1. Paired-sample T tests confirmed the overall efficacy of pioglitazone for improving insulin sensitivity (Table 2). Univariate ANCOVA demonstrated a significant interaction between childhood adversity and study group assignment in determining changes in plasma insulin response to glucose challenge after $120 \mathrm{~min}$, from beginning to end of the study (Table 3 ). Significant interactions were found between study group assignment and CTQ total, as well

Table 1

Characteristics of Study Subjects.

\begin{tabular}{|c|c|c|c|c|c|c|c|c|c|}
\hline \multicolumn{10}{|l|}{ Baseline Clinical Characteristics } \\
\hline \multirow[t]{2}{*}{ Variable } & \multicolumn{2}{|c|}{ Total Group $(\mathrm{N}=38)$} & \multicolumn{2}{|c|}{ Active Assignment $(\mathrm{N}=21)$} & \multicolumn{2}{|c|}{ Placebo Assignment $(\mathrm{N}=17)$} & \multirow[t]{2}{*}{ t-value } & \multirow[t]{2}{*}{ DF } & \multirow[t]{2}{*}{ p-value } \\
\hline & Mean & Std Dev & Mean & Std Dev & Mean & Std Dev & & & \\
\hline Age & 47.58 & 13.58 & 50.24 & 14.82 & 44.29 & 11.45 & 1.36 & 36 & 0.18 \\
\hline BMI & 30.45 & 6.26 & 30.34 & 6.17 & 30.60 & 6.58 & -0.12 & 34 & 0.90 \\
\hline Fasting Plasma Glucose & 96.56 & 11.16 & 96.38 & 12.84 & 96.79 & 9.05 & -0.11 & 36 & 0.91 \\
\hline Fasting Plasma Insulin & 13.70 & 6.06 & 14.03 & 5.64 & 13.29 & 6.70 & 0.37 & 36 & 0.71 \\
\hline HOMA & 3.29 & 1.54 & 3.35 & 1.45 & 3.21 & 1.67 & 0.27 & 36 & 0.79 \\
\hline $\begin{array}{l}\text { Oral Glucose Tolerance Test } \\
\text { (120 Min) }\end{array}$ & 109.19 & 27.09 & 102.39 & 20.76 & 117.59 & 31.98 & -1.77 & 36 & 0.09 \\
\hline Baseline HDRS-21 & 15.84 & 4.64 & 16.62 & 4.88 & 14.88 & 4.29 & 1.15 & 36 & 0.26 \\
\hline Medications & & & Active & ent & & Placebo & & & \\
\hline SSRI & & & 6 & & & 8 & & & \\
\hline SNRI & & & 6 & & & 1 & & & \\
\hline Bupropion & & & 1 & & & 3 & & & \\
\hline Mood Stabilizer & & & 5 & & & 6 & & & \\
\hline Antianxiety & & & 2 & & & 1 & & & \\
\hline Benzodiazepine & & & 3 & & & 2 & & & \\
\hline Sleeper & & & 3 & & & 2 & & & \\
\hline Stimulant & & & 0 & & & 3 & & & \\
\hline Antipsychotic & & & 3 & & & 2 & & & \\
\hline Mirtazapine & & & 1 & & & 0 & & & \\
\hline
\end{tabular}


Table 2

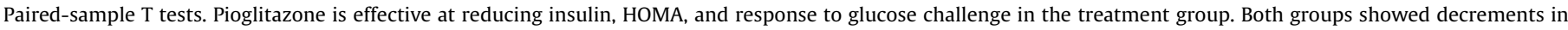
fasting glucose and in depressive symptoms, as previously published.

\begin{tabular}{|c|c|c|c|c|c|c|c|c|c|c|}
\hline \multicolumn{2}{|l|}{ Pioglitazone } & \multirow{3}{*}{ T2 Mean } & \multirow{3}{*}{ Mean Change } & \multirow{3}{*}{ Std. Dev } & \multirow{3}{*}{ Std. Error Mean } & & & \multirow[t]{2}{*}{$\mathrm{t}$} & \multirow[t]{2}{*}{ df } & \multirow[t]{2}{*}{ Sig. (2-tailed) } \\
\hline & T1 Mean & & & & & \multicolumn{2}{|c|}{$95 \% \mathrm{CI}$ of the Difference } & & & \\
\hline & & & & & & Lower & Upper & & & \\
\hline Glucose & 96.38 & 93.19 & 3.19 & 6.54 & 1.43 & 0.22 & 6.17 & 2.24 & 20 & 0.037 \\
\hline Fasting Insulin & 14.03 & 8.91 & 5.13 & 6.67 & 1.46 & 2.09 & 8.16 & 3.52 & 20 & 0.002 \\
\hline HOMA & 3.35 & 2.12 & 1.23 & 1.51 & 0.33 & 0.54 & 1.92 & 3.74 & 20 & 0.001 \\
\hline OGTT 30 & 148.50 & 136.28 & 12.22 & 32.53 & 7.10 & -2.59 & 27.02 & 1.72 & 20 & 0.101 \\
\hline OGTT 60 & 146.49 & 129.58 & 16.91 & 30.04 & 6.56 & 3.23 & 30.58 & 2.58 & 20 & 0.018 \\
\hline OGTT 90 & 128.83 & 115.16 & 13.68 & 26.51 & 5.78 & 1.61 & 25.74 & 2.36 & 20 & 0.028 \\
\hline OGTT 120 & 102.39 & 101.19 & 1.20 & 19.41 & 4.24 & -7.63 & 10.03 & 0.28 & 20 & 0.78 \\
\hline Ham-D & 16.62 & 13.19 & 3.429 & 3.4 & 0.74 & 1.88 & 4.98 & 4.62 & 20 & 0 \\
\hline \multicolumn{11}{|l|}{ Placebo } \\
\hline Glucose & 96.79 & 94.10 & 2.70 & 3.84 & 0.93 & 0.72 & 4.67 & 2.90 & 16 & 0.011 \\
\hline Fasting Insulin & 13.29 & 10.51 & 2.78 & 5.91 & 1.43 & -0.26 & 5.82 & 1.94 & 16 & 0.07 \\
\hline HOMA & 3.21 & 2.51 & 0.71 & 1.44 & 0.35 & -0.04 & 1.45 & 2.02 & 16 & 0.061 \\
\hline OGTT 30 & 143.18 & 141.73 & 1.45 & 20.08 & 4.87 & -8.87 & 11.77 & 0.30 & 16 & 0.77 \\
\hline OGTT 60 & 145.23 & 145.65 & -0.41 & 32.03 & 7.77 & -16.88 & 16.05 & -0.05 & 16 & 0.958 \\
\hline OGTT 90 & 133.94 & 132.60 & 1.34 & 24.24 & 5.88 & -11.12 & 13.81 & 0.23 & 16 & 0.822 \\
\hline OGTT 120 & 117.59 & 117.13 & 0.46 & 23.26 & 5.64 & -11.50 & 12.42 & 0.08 & 16 & 0.936 \\
\hline Ham-D & 14.88 & 12.06 & 2.82 & 3.05 & 0.74 & 1.26 & 4.39 & 3.82 & 16 & 0.002 \\
\hline
\end{tabular}

as separately for all CTQ subscales excepting physical neglect, in predicting changes in insulin secretion between the start and end of the study. No significant interactions were found for the outcomes of change in fasting glucose or glucose response to OGTT, or for changes in depression scores, although there was a trend for higher glucose response peaks in the OGTT among individuals with trauma histories. We performed a parallel analysis using a repeated-measures ANCOVA that covered all four time points in the glucose challenge test (0,60 min, $90 \mathrm{~min}$, and $120 \mathrm{~min})$, and found substantially similar results (not shown).

\subsection{Direction of association between childhood adversity and changes in mood and metabolism}

Higher rates of childhood adversity (physical abuse, physical neglect, emotional abuse, and total CTQ) were associated with

Table 3

Univariate ANCOVAs reveal significant interactions between measures of childhood adversity and study group assignment in change in insulin secretion in response to pioglitazone. Significant effects in bold type.

\begin{tabular}{|c|c|c|c|}
\hline & $\mathrm{F}$ & Mean Square & Sig \\
\hline CTQ total & 4.845 & 6,231 & 0.034 \\
\hline Study group & 5.044 & 6,486 & 0.031 \\
\hline CTQ*Study group & 4.348 & 5,910 & 0.021 \\
\hline Emotional Abuse & 5.280 & 6,717 & 0.028 \\
\hline Study group & 5.443 & 6,294 & 0.026 \\
\hline Emotional Abuse*Study group & 4.779 & 6,371 & 0.015 \\
\hline Physical Abuse & 7.182 & 8,724 & 0.011 \\
\hline Study group & 4.941 & 6,002 & 0.033 \\
\hline Physical Abuse*Study group & 6.099 & 7,676 & 0.005 \\
\hline Sexual Abuse & 0.212 & 308 & 0.648 \\
\hline Study group & 5.782 & 8,414 & 0.022 \\
\hline Sexual Abuse*Study group & 3.749 & 5,241 & 0.033 \\
\hline Emotional Neglect & 0.533 & 769 & 0.470 \\
\hline Study group & 5.371 & 7,745 & 0.026 \\
\hline Emotional Neglect*Study group & 1.420 & 2,229 & 0.255 \\
\hline Physical Neglect & 3.070 & 4,132 & 0.089 \\
\hline Study group & 4.556 & 6,132 & 0.040 \\
\hline Physical Neglect*Study group & 3.142 & 4,520 & 0.056 \\
\hline
\end{tabular}

increases in fasting plasma insulin after twelve weeks of treatment with pioglitazone (Fig. 1). Thus, the overall positive response to pioglitazone was driven by individuals without histories of childhood adversity. Additionally, the increased resistance to pioglitazone conferred by childhood adversity was not simply a function of greater baseline insulin resistance, as none of baseline fasting glucose, fasting insulin, or HOMA correlated significantly with the insulin response to pioglitazone treatment.

\subsection{Insulin resistance at baseline}

We also performed an additional analysis examining changes in depressive symptoms, only among those patients who had insulin resistance at baseline $(\mathrm{N}=20$, of which 13 received pioglitazone and 7 received placebo). This was based on our previous finding that pioglitazone improved depressive symptoms only among those with insulin resistance at baseline [13]. While the interaction between study group assignment and CTQ score was not significant in this limited sample $(F=4.71$, mean square $=16.09$, $\mathrm{p}=0.072$ ), the placebo group showed a trend towards less improvement in depressive symptoms with higher CTQ scores, whereas the pioglitazone group showed no such trend (Fig. 2). No interaction was found between study group assignment and CTQ score with respect to changes in depressive symptoms among the insulin sensitive group $(p=0.931)$.

\section{Conclusions and discussion}

Our finding that childhood adversity impairs metabolic response to pioglitazone highlights the potential that early life adversity may have far-reaching negative effects on physiology over the lifespan by interfering with the body's ability to respond to available treatments.

\subsection{Multiple subtypes of childhood adversity contribute to metabolic resistance to pioglitazone}

While only emotional abuse was associated with baseline metabolic factors, metabolic changes in response to pioglitazone were significantly affected by total CTQ as well as by the subscales of emotional abuse, physical abuse, and physical neglect. The use of a randomized intervention created a larger separation between the 
Change in insulin release at 120 minutes post glucose

challenge after twelve weeks of pioglitazone treatment

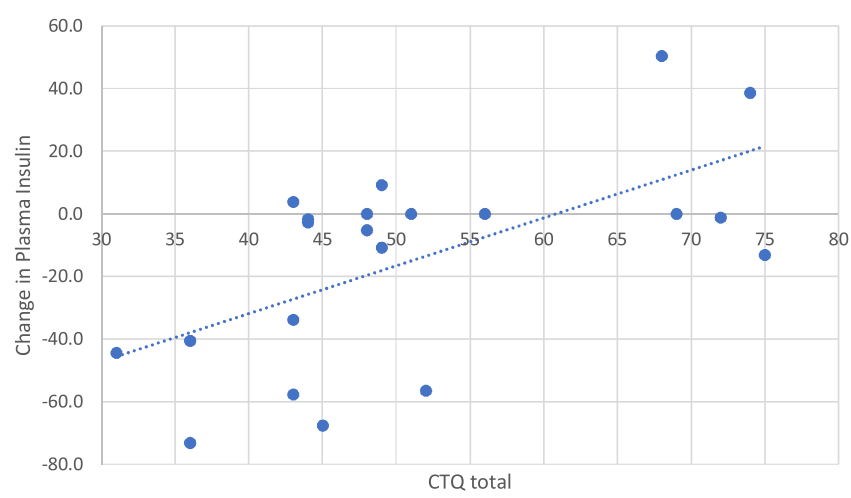

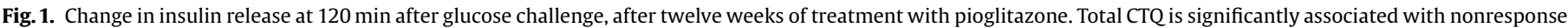
to pioglitazone by repeated-measures ANOVA on the pioglitazone group only, $\mathrm{N}=21$. $(\mathrm{F}=11.539$, mean $\mathrm{square}=6714$, $\mathrm{p}=0.003)$.

control and pioglitazone populations, likely amplifying our ability to detect the effect of interest. While it is quite likely that distinct subtypes of childhood adversity operate on metabolism via distinct mechanisms, it is also possible that the differences we found among CTQ subscales represent stochastic variation related to small sample size. Larger studies of this type would be needed to sort out these questions.

While we examined changes in fasting glucose and oral glucose tolerance as well, insulin response to glucose load was the only outcome that was clearly affected by childhood adversity. It may be that fasting insulin represents a 'cleaner' outcome, one more heavily determined by internal biological factors and less susceptible to perturbation by recent, short-term environmental effects than serum glucose.

\subsection{Effects of baseline insulin resistance on response to treatments for depression}

This trial was originally designed to investigate whether pioglitazone could facilitate response to standard treatments for depression. The treatment and control groups overall did not separate on our depression measures: all individuals received treatment as usual for depression, and depressive symptoms fell significantly in both groups. In the initial study, a model that included baseline insulin resistance revealed that only insulin resistant subjects benefited from pioglitazone augmentation for depression [13]. We therefore repeated our analysis in the current study while limiting our sample pool to only those participants with baseline insulin resistance. However, because we were examining an interaction with a covariate, our study power was more limited, and thus the further power reduction imposed by limiting the analysis to insulin resistant individuals brought any effects below the limit of detection.

Nonetheless, the observed trends indicated that among those insulin resistant participants who received placebo, higher CTQ scores were associated with continuing depression over the course of the study, whereas among insulin resistant participants who received pioglitazone, depression tended to improve regardless of CTQ score (Fig. 2). These trends are consistent with the hypothesis that childhood adversity confers a combined phenotype of depression and insulin resistance, which is partially reversed by insulin sensitizing medication, permitting depressed, previously

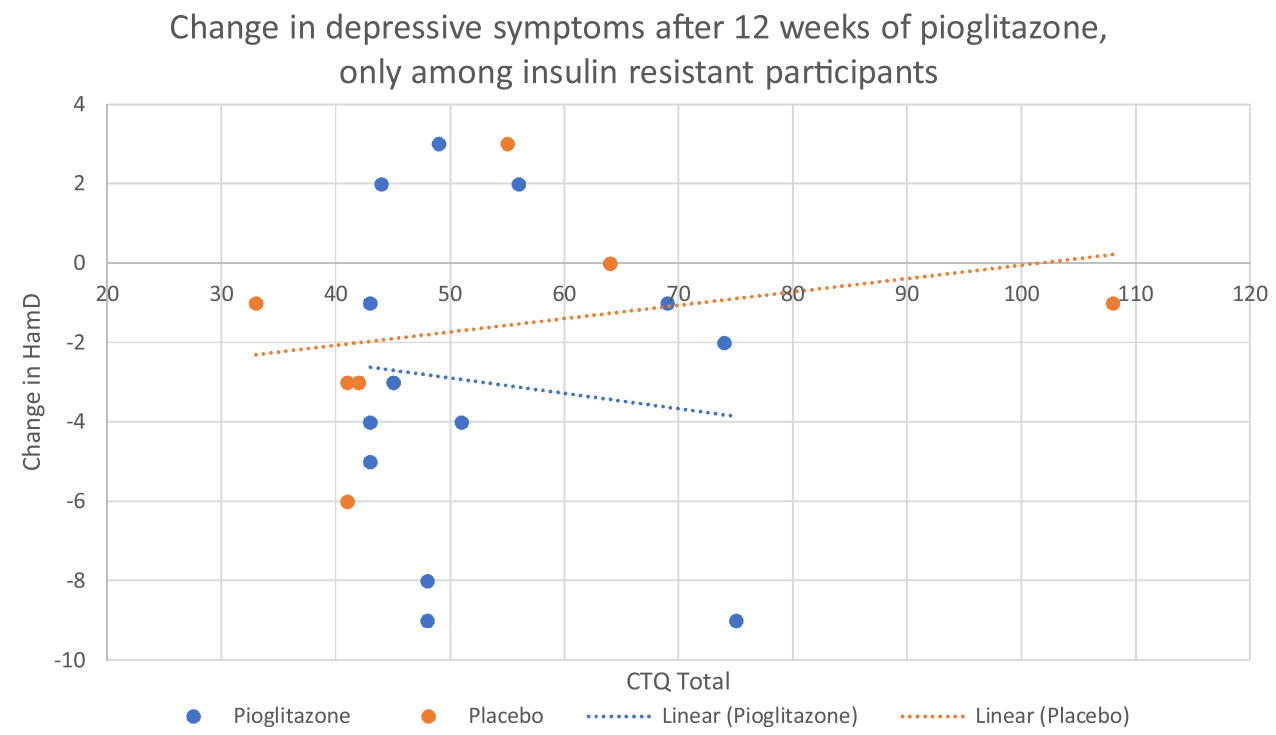

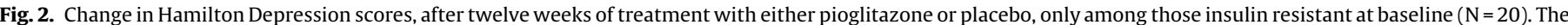

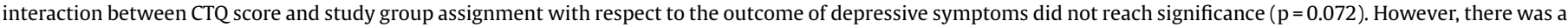
trend for less improvement in mood with increasing CTQ score only in the placebo group, whereas no such trend was evident for the pioglitazone group. 
insulin resistant individuals to respond more effectively to standard treatments for depression. It is possible that if we had a larger sample of insulin resistant individuals, this observed trend for an interaction between childhood adversity and study group assignment for the outcome of depressive symptoms could have reached significance.

Early life adversity has long been known to confer resistance to standard psychopharmacological treatments for depression [8]. Our work suggests that metabolic dysfunction could be a mediating factor in the relationship between childhood adversity and resistance to treatment for depression.

\subsection{Allostatic load as a determinant of responsiveness to treatment}

Our results suggest that early life adversity confers a lasting metabolic change that impairs responsiveness to pioglitazone, certainly from a metabolic perspective and perhaps also from a psychiatric perspective. These results represent an interesting parallel to a previously published analysis of this cohort, which revealed that telomere length, another indicator of allostatic load, was positively associated with the antidepressant response to pioglitazone [18]. Telomere length was not, however, associated with the antidiabetic response to pioglitazone in that analysis. This suggests that allostatic load may include a number of different components that interact with PPAR $y$ agonist action through distinct mechanisms to affect mood and metabolism. The component of allostatic load reflected by telomere length may be most relevant to mood symptoms, while the component reflected by childhood adversity score may be more highly relevant to metabolic symptoms. As PPAR $\gamma$ is a transcription factor whose activation initiates multiple parallel downstream cascades, it is quite possible that the aspect of PPAR $\gamma$ action that interacts with telomere length to affect mood may be distinct from the aspect that interacts with childhood adversity score to affect metabolism.

\subsection{Potential mechanisms}

We speculate that increased levels of inflammation could have mediated the effects observed in this study. Insulin resistance, childhood trauma history, and depression are known to have overlapping and related inflammatory components. Type II diabetes and depression share a common molecular signature of increased inflammation, involving elevation of C-reactive protein (CRP), tumor necrosis factor alpha (TNF $\alpha$ ), and interleukin-6 (IL6), and possibly depression of transforming growth factor beta (TGF $\beta$ ) and adiponectin [19-22]. Childhood trauma is also known to be associated with proinflammatory phenotypes in adulthood, including elevation of CRP and TNF $\alpha$, and possibly also IL-6 [23].

PPAR $\gamma$ is known to affect insulin sensitivity via pleiotropic mechanisms, including glucose homeostasis, lipid metabolism, and adipokine regulation [24]. Adipokine mediators of PPAR $\gamma$ action include adiponectin, TNF $\alpha$, and IL-6, all of which have been repeatedly associated with depressive symptoms. Additionally, animal work has shown that the antidepressant-like effects of pioglitazone are associated with PPAR $\gamma$-mediated reduction in inflammatory cytokines, including expression of TNF $\alpha$, and IL-6, in murine hippocampal microglia [26].

It is thus possible that individuals with histories of childhood trauma have constitutively higher levels of TNF $\alpha$, IL-6, and perhaps other relevant inflammatory mediators, and that chronic high levels of inflammatory signaling result in lowered sensitivity to the perturbations in these pathways induced by PPAR $\gamma$ activation. Thus people without childhood trauma histories and with constitutively low levels of inflammatory mediators may display greater responsiveness to the alterations in these pathways produced by pioglitazone treatment, whereas individuals with trauma histories who express higher levels of the above inflammatory mediators may have downregulated the relevant receptors in these signaling pathways, and thus be less susceptible to pioglitazone-induced alterations in ligand levels.

Unfortunately we did not have any direct data on inflammatory markers in the current study cohort that would help to support or disprove the above hypothesis.

\subsection{Limitations}

The main limitation of this study was its small sample size. This is the likely explanation why we did not detect broader associations between early life adversity and adult metabolic impairment and depression, and also posed a limitation to our ability to stratify by baseline insulin resistance, which would likely have yielded further important insights. As this was among the earliest randomized controlled trials of metabolic sensitizing agents for depression, our results can be used to estimate effect sizes for future, more appropriately powered studies. The randomized interventional design was the study's greatest strength, as individual differences in treatment response are very difficult to detect in observational investigations.

\subsection{Future directions}

Our findings have clinical importance in that they indicate that patients with metabolic dysfunction and histories of childhood adversity may be less likely to respond to antidiabetic medications. Intriguingly, they also suggest that among individuals with both insulin resistance and depression, insulin-sensitizing treatment may disrupt the association between childhood adversity and poor response to treatment for depression.

Larger and more effectively powered studies are needed to confirm these results. If confirmed, our results could indicate that assessment of childhood history may assist clinicians in identifying patients who may be at risk for treatment-resistant diabetes. Future investigations would then be needed to identify methods for overcoming such treatment resistance.

\section{Author statement}

Thalia Robakis conceived of the question, analyzed the data, and wrote the paper, with input and assistance from other authors. Kathleen Watson provided statistical oversight and conceptual input. Alison Myoraku assisted with data processing, construction of tables, and editing and formatting of references. Tonita Wroolie obtained the CTQ data from study participants. Carla Nasca, Benedetta Bigio, and Bruce McEwen contributed conceptual input and comments/editing on the manuscript. Natalie Rasgon conceived of, funded, and conducted the original parent study and maintains proprietorship of the data, and also contributed conceptual input to this analysis.

\section{Conflict ofinterest}

Dr. Rasgon has received research support from Corcept Pharmaceuticals, Bayer, Abbot, Forest Laboratories, GlaxoSmithKline, Pfizer, and Magceutics, and has received consultant or speaker fees from Takeda, Shire, Sunovion, Bristol-Myers Squibb, Pfizer, and Wyeth. Drs. Robakis, Watson, Wroolie, Myoraku, Nasca, Bigio, and McEwen have nothing to disclose.

\section{Funding}

Funding for this research was provided by the National Institutes of Mental Health grant 1R21 MH093948-01A1, to principal investigator 


\section{Dr. Rasgon. Drs. Nasca, Bigio, and McEwen were also supported by the Hope for Depression Research Foundation.}

\section{References}

[1] Campbell J.A., Farmer GC, Nguyen-Rodriguez S, Walker R, Egede L. Relationship between individual categories of adverse childhood experience and diabetes in adulthood in a sample of US adults: Does it differ by gender? J Diabetes Complicat 2018;32(2):139-43.

[2] Chapman DP, Whitfield CL, Felitti VJ, Dube SR, Edwards VJ, Anda RF. Adverse childhood experiences and the risk of depressive disorders in adulthood. J Affect Disord 2004;82(2):217-25.

[3] Mezuk B, Eaton WW, Albrecht S, Golden SH. Depression and type 2 diabetes over the lifespan. Diabetes Care 2008;31(12):2383-90.

[4] Danese A, Moffitt TE, Harrington H, Milne BJ, Polanczyk G, Pariante CM, et al. Adverse childhood experiences and adult risk factors for age-related disease: depression, inflammation, and clustering of metabolic risk markers. Arch Pediatr Adolesc Med 2009;163(12):1135-43.

[5] Manolopoulos VG, Ragia G, Tavridou A. Pharmacogenomics of oral antidiabetic medications: current data and pharmacoepigenomic perspective. Pharmacogenomics 2011;12(8):1161-91.

[6] Stratton IM, Adler AI, Neil HAW, Matthews DR, Manley SE, Cull CA, et al Association of glycaemia with macrovascular and microvascular complications of type 2 diabetes (UKPDS 35): prospective observational study. Bmj 2000;321 (7258):405-12.

[7] Souery D, Papakostas GI, Trivedi MH. Treatment-resistant depression. J Clin Psychiatry 2006;67:16-22.

[8] Nemeroff CB, Heim CM, Thase ME, Klein DN, Rush AJ, Schatzberg AF, et al. Differential responses to psychotherapy versus pharmacotherapy in patients with chronic forms of major depression and childhood trauma. Proc Natl Acad Sci 2003;100(24):14293-6.

[9] Pace TW, Hu F, Miller AH. Cytokine-effects on glucocorticoid receptor function: relevance to glucocorticoid resistance and the pathophysiology and treatment of major depression. Brain Behav Immun 2007;21(1):9-19.

[10] Rasgon NL, Kenna HA, Williams KE, Powers B, Wroolie T, Schatzberg AF. Rosiglitazone add-on in treatment of depressed patients with insulin resistance: a pilot study. Sci World J 2010;10:321-8.

[11] Kemp DE, Ismail-Beigi F, Ganocy SJ, Conroy C, Gao K, Obral S, et al. Use of insulin sensitizers for the treatment of major depressive disorder: a pilot study of pioglitazone for major depression accompanied by abdominal obesity. J Affect Disord 2012;136(3):1164-73.
[12] Kashani L, Omidvar T, Farazmand B, Modabbernia A, Ramzanzadeh F, Tehraninejad ES, et al. Does pioglitazone improve depression through insulin-sensitization? Results of a randomized double-blind metformincontrolled trial in patients with polycystic ovarian syndrome and comorbid depression. Psychoneuroendocrinology 2013;38(6):767-76.

[13] Lin KW, Wroolie TE, Robakis T, Rasgon NL. Adjuvant pioglitazone for unremitted depression: clinical correlates of treatment response. Psychiatry Res 2015;230(3):846-52.

[14] Berger J, Moller DE. The mechanisms of action of PPARs. Annu Rev Med 2002;53(1):409-35.

[15] Shea M, Nicklas BJ, Marsh AP, Houston DK, Miller GD, Isom S, et al. The effect of pioglitazone and resistance training on body composition in older men and women undergoing hypocaloric weight loss. Obesity 2011;19(8):1636-46.

[16] Shadid S, Jensen MD. Effects of pioglitazone versus diet and exercise on metabolic health and fat distribution in upper body obesity. Diabetes Care 2003;26(11):3148-52.

[17] Bernstein DP, Stein JA, Newcomb MD, Walker E, Pogge D, Ahluvalia T, et al. Development and validation of a brief screening version of the Childhood Trauma Questionnaire. Child Abuse Negl 2003;27(2):169-90.

[18] Rasgon N, Lin KW, Lin J, Epel E, Blackburn E. Telomere length as a predictor of response to Pioglitazone in patients with unremitted depression: a preliminary study. Transl Psychiatry 2016;6(1):e709.

[19] Furtado M, Katzman MA. Examining the role of neuroinflammation in major depression. Psychiatry Res 2015;229(1):27-36.

[20] Dowlati Y, Herrmann N, Swardfager W, Liu H, Sham L, Reim EK, et al. A metaanalysis of cytokines in major depression. Biol Psychiatry 2010;67(5):446-57.

[21] Stuart MJ, Baune BT. Depression and type 2 diabetes: inflammatory mechanisms of a psychoneuroendocrine co-morbidity. Neurosci Biobehav Rev 2012;36(1):658-76.

[22] Liu J, Guo M, Zhang D, Cheng SY, Liu M, Ding J, et al. Adiponectin is critical in determining susceptibility to depressive behaviors and has antidepressantlike activity. Proc Natl Acad Sci 2012;109(30):12248-53.

[23] Baumeister D, Akhtar R, Ciufolini S, Pariante CM, Mondelli V. Childhood trauma and adulthood inflammation: a meta-analysis of peripheral C-reactive protein, interleukin-6 and tumour necrosis factor- $\alpha$. Mol Psychiatry 2016;21 (5):642.

[24] Choi SS, Park J, Choi JH. Revisiting PPAR $\gamma$ as a target for the treatment of metabolic disorders. BMB Rep 2014;47(11):599.

[26] Zhao Q, Wu X, Yan S, Xie X, Fan Y, Zhang J, et al. The antidepressant-like effects of pioglitazone in a chronic mild stress mouse model are associated with PPAR $\gamma$-mediated alteration of microglial activation phenotypes. J Neuroinflammation 2016;13(1):259. 